\title{
Rare and Exotic Higgs decays, including new scalars
}

\author{
Linda Finco*, on behalf of the ATLAS and CMS Collaborations \\ University of Nebraska - Lincoln \\ E-mail: linda.fincodcern.ch
}

\begin{abstract}
The latest results in the search for rare and exotic Higgs boson decays are presented. The analyses are performed using proton-proton collision data collected with the ATLAS and CMS detectors at the LHC at a centre-of-mass energy $\sqrt{s}=13 \mathrm{TeV}$. Several decay modes are reviewed, including very rare Standard Model decays as well as decays not predicted by this theory, such as lepton-flavour-violating decays and decays to non-Standard-Model particles. No evidence for new physics is found and the results are interpreted to set limits on the cross section times branching fraction of such decays.
\end{abstract}

7th Annual Conference on Large Hadron Collider Physics - LHCP2019

20-25 May, 2019

Puebla, Mexico

${ }^{*}$ Speaker. 


\section{Introduction}

The discovery of a new particle consistent with the Standard Model (SM) Higgs boson [1, 2, 3] has completed the theory, shedding light on one of the most important questions of physics: the origin of the mass of elementary particles. Nevertheless, the SM cannot address several crucial issues, both based on direct evidence from observation, such as the neutrino masses, the existence of dark matter and dark energy, or the matter-antimatter asymmetry, and conceptual problems in the SM itself, such as the large number of free parameters, the "hierarchy problem" and the coupling unification. There are thus strong indications that the SM is only a low-energy expression of a more global theory.

The search for physics beyond the SM (BSM) is one of the main goals of the LHC, in particular in the Higgs sector. The SM Higgs boson has indeed a very narrow width $(\sim 4 \mathrm{MeV})$ and the current measurements still allow for additional contributions from BSM decays, setting an indirect limit on the Higgs boson branching fraction to BSM particles at $0.34(\mathscr{B}(H \rightarrow B S M)<34 \%)$ [4]. Two main possibilities can be exploited to detect new physics in the SM scalar sector: by looking for a direct evidence through the observation of BSM decays of the Higgs boson and by searching for indirect hints through the measurement of deviations in its couplings. Given the limited precision of the latter approach, only the former possibility will be described in this note.

The direct search for BSM physics in the Higgs sector can be investigated through Higgs boson decays to both SM particles (such as very rare SM decays and decays forbidden by the SM), and to particles not predicted by the SM (like Higgs boson decays to dark matter candidates or to light pseudoscalars/scalars). In the following, the latest results in the search for rare and exotic Higgs boson decays using proton-proton (pp) collision data collected by the ATLAS [5] and CMS [6] experiments at a centre-of-mass energy $\sqrt{s}=13 \mathrm{TeV}$ are summarized.

\section{Rare Decays}

The SM predicts many decay modes for the Higgs boson. In order to characterize its properties in a comprehensive way, it is important to look at all the possible decay channels, some of which, with very small branching fraction, have yet to be observed. Several BSM theories predict substantial modifications to the direct couplings of the Higgs boson to fermions, which can enhance the branching fraction of rare decays with respect to SM predictions, allowing them to be seen even with the current dataset.

The latest results on rare decays of the Higgs boson carried out at the $\mathrm{LHC}^{1}$ concern the search for $H \rightarrow J / \psi \gamma$ and $H \rightarrow \Upsilon \gamma$, as well as $H \rightarrow J / \psi J / \psi$ and $H \rightarrow \Upsilon \Upsilon$ processes. These decay channels are a promising laboratory to study Yukawa couplings, in particular to light quarks, which are still not directly observed. In both analyses, a potential signal would be visible as a narrow bump in the mass distribution of the decay particles over a smoothly falling background. Table 1 reports the branching fractions of the aforementioned processes as predicted by the SM.

Searches for the exclusive decays of the Higgs boson into a $J / \psi, \psi(2 S)$, or $\Upsilon(n S)$ (with $n=1,2$, $3)$ meson and a photon have been performed by the ATLAS collaboration, using a pp collision data sample corresponding to an integrated luminosity $\mathscr{L}=36.1 \mathrm{fb}^{-1}$, collected at $\sqrt{s}=13 \mathrm{TeV}$ [7].

\footnotetext{
${ }^{1}$ At the time of the conference.
} 


\begin{tabular}{ll}
\hline Decay Channel & Branching Fraction \\
\hline$H \rightarrow J / \psi \gamma$ & $\sim 3 \times 10^{-6}$ \\
$H \rightarrow \Upsilon \gamma$ & $\sim 5 \times 10^{-9}$ \\
$H \rightarrow \Upsilon$ & $\sim 2 \times 10^{-9}$ \\
$H \rightarrow J / \psi J / \psi$ & $\sim 1.5 \times 10^{-10}$ \\
\hline
\end{tabular}

Table 1: Branching fractions of the rare Higgs boson decay channels described in this note as predicted by the Standard Model.

The analysis focuses on the clean di-muon final state and targets high-rate inclusive Higgs and $\mathrm{Z}$ bosons production. In Figure 1 (left) the mass of the $\mu^{+} \mu^{-} \gamma$ system of the $\Upsilon(n S) \gamma$ candidates selected in the barrel is represented in both data and simulation. Given that no significant excess of events is observed above the background expectations in any of the channels under study, $95 \%$ confidence level (CL) upper limits are extracted and are able to exclude branching fraction down to $10^{-4}\left(\mathscr{B}(H \rightarrow J / \psi \gamma)<3.5 \times 10^{-4}, \mathscr{B}(H \rightarrow \psi(2 S) \gamma)<2.0 \times 10^{-3}\right.$ and $\mathscr{B}(H \rightarrow J / \Upsilon(n S) \gamma)<$ $\left.(4.9,5.9,5.7) \times 10^{-4}\right)$.

The decay of the Higgs boson to pairs of $J / \psi$ or $\Upsilon(n S)(n=1,2,3)$ has been investigated for the first time by the CMS collaboration, analysing data from pp collisions collected at $\sqrt{s}=13 \mathrm{TeV}$ in $2017\left(\mathscr{L}=37.5 \mathrm{fb}^{-1}\right)$ [8]. The four-muon final state offers a very clean experimental signature with relatively narrow intermediate resonant states and benefits of dedicated triggers deployed to enhance it. Figure 1 (right) shows the four-muon invariant mass distribution in the $J / \psi J / \psi$ final state. Given the absence of a signal for either of the decay channels, upper limits on the branching fractions are obtained and are set at $\mathscr{B}(H \rightarrow J / \psi J / \psi)<1.8 \times 10^{-3}$ and $\mathscr{B}(H \rightarrow \Upsilon \Upsilon)<1.4 \times$ $10^{-3}$.
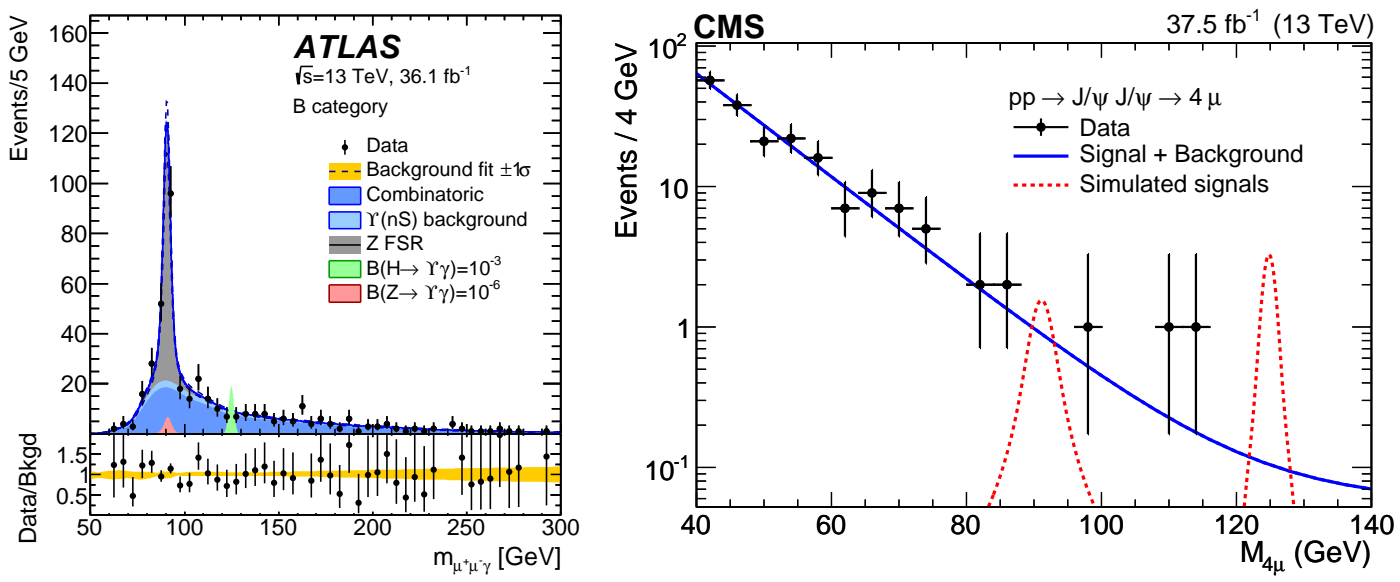

Figure 1: Left: The $m_{\mu^{+} \mu^{-} \gamma}$ distribution for the selected $\Upsilon(n S) \gamma$ candidates detected in the barrel of the ATLAS experiment along with the results of the maximum-likelihood fits with background-only models [7]. Right: The four-muon invariant mass distribution, for $J / \psi J / \psi$ candidates, with superimposed maximum likelihood fit (solid blue line) [8]. 


\section{Lepton Flavour Violating Decays}

A possible sign of new physics is lepton flavour violation in decays of the Higgs boson, which is forbidden by the SM. Many searches have been carried out on this subject at the LHC [9, 10, 11, 12], the most recent one being the study of $H \rightarrow e \tau$ and $H \rightarrow \mu \tau$ decays performed by the ATLAS collaboration [13]. This analysis uses pp collisions at $\sqrt{s}=13 \mathrm{TeV}$ with an integrated luminosity $\mathscr{L}=36.1 \mathrm{fb}^{-1}$. It is performed in four signal regions, according to the $\tau$ decay (leptonic or hadronic) and the Higgs boson production mode. Boosted decision tree algorithms are used to enhance the signal separation from background events. In the absence of any significant excess, upper limits on the branching ratios are derived. The observed (expected) 95\% CL upper limits are $0.47 \%\left(0.34_{-0.10}^{+0.13} \%\right)$ and $0.28 \%\left(0.37_{-0.10}^{+0.14} \%\right)$ for the $H \rightarrow e \tau$ and $H \rightarrow \mu \tau$ searches, respectively. These limits are significantly lower than the corresponding Run 1 limits. Similar results have been obtained by the CMS collaboration [14].

\section{Decays to Invisible Particles}

Another possibility to search for BSM decays of the Higgs boson is to probe its decays to non-SM particles, like dark matter (DM) candidates, that could be invisible to our detectors. In the SM, the Higgs boson decays invisibly only through the $H \rightarrow Z Z \rightarrow 4 v$ process, with a branching fraction $\mathscr{B}(H \rightarrow$ inv $)$ of about $10^{-3}$. The rate for invisible decays may be significantly enhanced in the context of several BSM scenarios, where the $125 \mathrm{GeV}$ boson is a portal between a dark sector and the SM sector. Given that DM particles escape detection, Higgs boson decays to them are characterized by large missing transverse momentum. All the main Higgs production modes can be used to probe Higgs boson couplings to dark matter and the Higgs boson recoil against a visible system is employed to distinguish among them. The most sensitive mode is vector boson fusion (VBF), where the Higgs boson is produced along with two jets with a large separation in pseudorapidity and a large di-jet invariant mass, a characteristic signature which allows for the suppression of SM backgrounds.

Both the ATLAS [15] and CMS [16] collaborations performed a combination of the different production modes of 2016 measurements, assuming SM production cross sections, as well as a combination of Run 1 and Run 2 analyses. They have both investigated VBF and associated production with a vector boson, while only CMS has so far performed studies on gluon fusion and associated production with a top pair [17]. Figure 2 (left and middle) represents the results of the Run 1 and Run 2 combination for both experiments: current observed (expected) 95\% CL upper limits on $\left(\sigma / \sigma_{S M}\right) B(H \rightarrow i n v)$ are $0.26(0.17)$ and $0.19(0.15)$ for ATLAS and CMS, respectively.

Another search for invisible particles has been performed by CMS [18], investigating the process where the Higgs boson is produced in association with a $\mathrm{Z}$ boson and decays to an invisible particle (a massless dark photon) together with an isolated photon. This analysis uses a dataset recorded in 2016-18 corresponding to an integrated luminosity of $137.4 \mathrm{fb}^{-1}$, at a centre-of-mass energy of 13 $\mathrm{TeV}$. The final state is characterized by large missing transverse energy (due to the dark photon), two opposite-sign and same-flavour leptons (coming from the $\mathrm{Z}$ boson) and a photon. No significant excess of events above the SM expectation is found, and the results are interpreted to place limits on the cross section times branching fraction of such decay. The observed and expected 
95\% CL upper limits assuming the SM production rate on $\mathscr{B}(H \rightarrow i n v+\gamma)$ are $4.6 \%$ and $3.6 \%$, respectively, and are reported in Figure 2 (right).
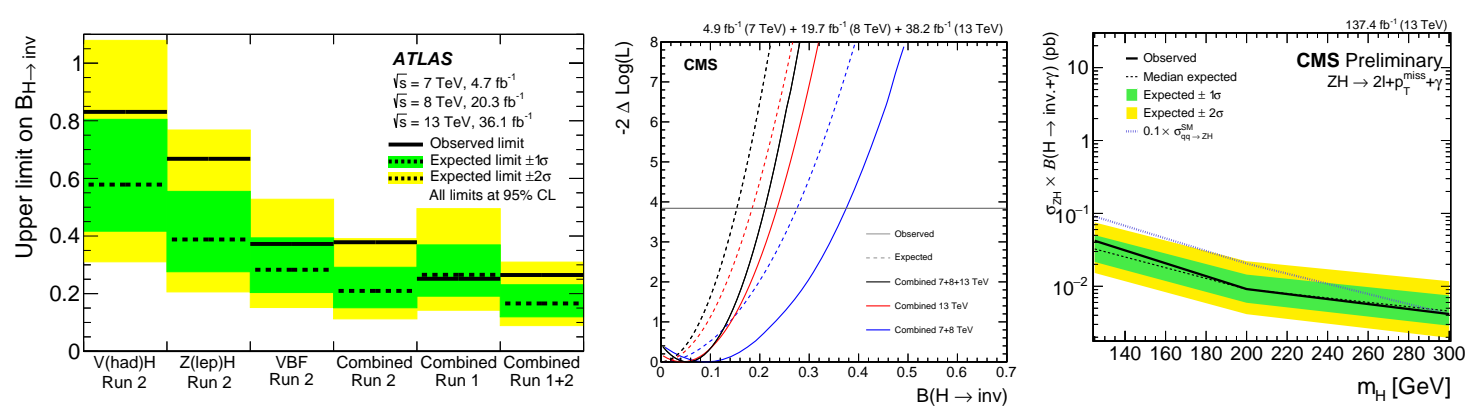

Figure 2: Left: The observed and expected upper limits on $\mathscr{B}(H \rightarrow$ inv $)$ at $95 \%$ CL from direct searches for invisible decays of the $125 \mathrm{GeV}$ Higgs boson and their statistical combinations in Run 1 and Run 2 measured by ATLAS [15]. Middle: Profile likelihood ratios as a function of $\mathscr{B}(H \rightarrow i n v)$ obtained by CMS [16]. The solid curves represent the observations in data, while the dashed lines represent the expected result obtained from the background-only hypothesis. Right: Expected and observed 95\% CL upper limits on $\sigma_{Z H} \times \mathscr{B}(H \rightarrow i n v+\gamma)$ as a function of $m_{H}$ measured by CMS [18]. The expected $\sigma_{Z H}$ assuming $\mathscr{B}(H \rightarrow$ $i n v+\gamma)=10 \%$ is also shown.

\section{Exotic Decays}

The last possibility analysed in this note to probe new physics effects is to consider extensions of the SM scalar sector, as done in the Two-Higgs-Doublets Models (2HDMs). These models introduce two doublets of scalar fields $\left(\phi_{1}\right.$ and $\left.\phi_{2}\right)$ in the SM lagrangian, which, after symmetry breaking, lead to five physical states: two charged scalars, one CP-odd pseudoscalar and two neutral scalars. According to how $\phi_{1}$ and $\phi_{2}$ couple with quarks and leptons, four 2HDM types can be defined. A further extension of $2 \mathrm{HDMs}$, where a complex scalar singlet is added to the already present scalar doublets, is called $2 \mathrm{HDM}+\mathrm{S}$ and introduces the additional (pseudo)scalar $a$, opening up the possibility to investigate $H \rightarrow a a$ exotic decays, while still being consistent with all the LHC measurements so far.

The main final states into which $a$ could decay are $a \rightarrow b \bar{b}, a \rightarrow \tau^{+} \tau^{-}$and $a \rightarrow \mu^{+} \mu^{-}$. The first two, when kinematically accessible, have a large branching ratio (if SM couplings are supposed), but they are more difficult to trigger and have a low identification efficiency. The last channel, instead, has a very clean signature, but an extremely low branching fraction. Moreover, depending on the value of the $a$ mass, decay products can have a different topology. Below $m_{a} \sim 15 \mathrm{GeV}$, given the large difference in mass between $a$ and the Higgs boson, the $a$ bosons will be produced with high Lorentz boost and their decay products will be strongly collimated. In this case, special boosted reconstruction techniques have to be used. On the other hand, for $m_{a}$ above $\sim 15 \mathrm{GeV}$, the final state particles are well separated and the usual reconstruction techniques are applied. Several searches for $H \rightarrow a a$ decays have been performed by the ATLAS and CMS collaborations using Run 1 and Run 2 LHC data, exploiting various decay modes of the $a$ boson and probing different ranges of its mass. The results depend on the model type considered and on the $\tan (\beta)$ parameter, 
i.e. the ratio of the vacuum expectation values of the two additional Higgs doublets. As an example, results for a $2 \mathrm{HDM}+\mathrm{S}$ type II model (for $\tan (\beta)=2$ ) are summarized in Figure 3, for both ATLAS (left) and CMS (right) collaborations.

The latest result published by the CMS collaboration on Higgs exotic decays is the search for an
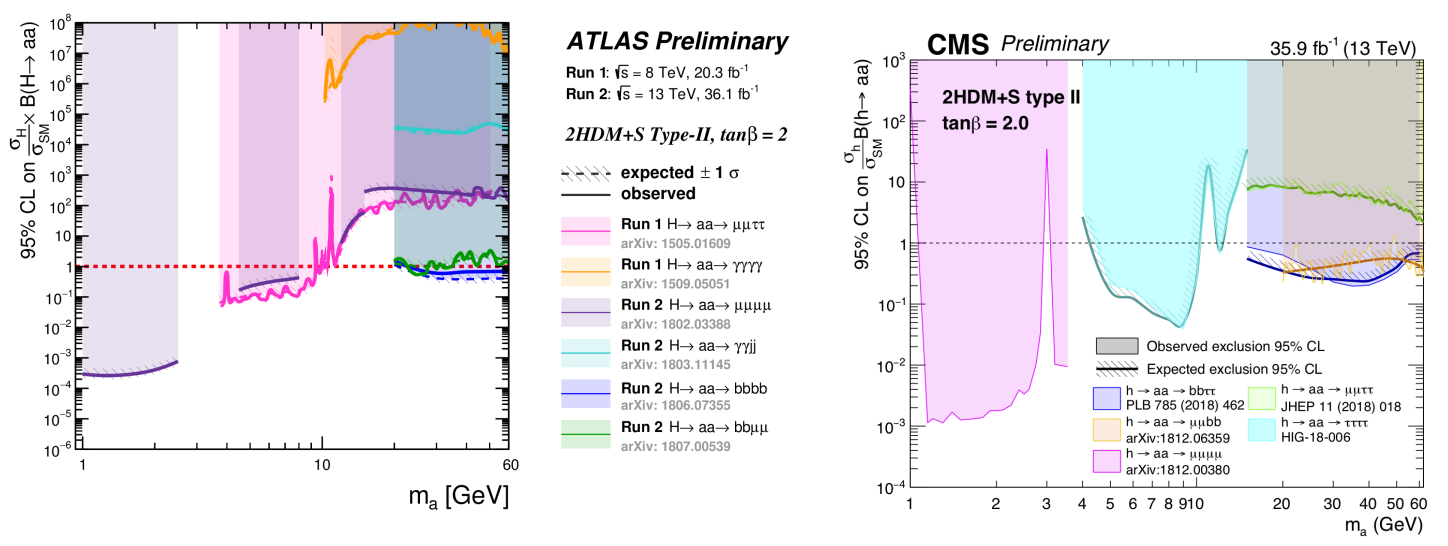

Figure 3: $95 \% \mathrm{CL}$ on $\sigma(H) / \sigma_{S M} \times \mathscr{B}(H \rightarrow a a)$ in the $2 \mathrm{HDM}+\mathrm{S}$ type- $2 \tan (\beta)=2$ scenario for exotic $\mathrm{H}$ decay searches performed by ATLAS (left) [19] and CMS (right) [20] with data collected at $13 \mathrm{TeV}$ center-of-mass energy.

highly-boosted $a$ boson, with a mass between 4 and $15 \mathrm{GeV}$, decaying into two muons and two nearby tracks [21]. This analysis uses pp collision data corresponding to $\mathscr{L}=35.9 \mathrm{fb}^{-1}$, collected at $13 \mathrm{TeV}$. It employs special techniques to select and identify Lorentz-boosted lepton pairs with overlapping decay products. No evidence of signal is found in data, thus upper limits are extracted and are represented in Figure 4 (left). The sensitivity of the search is reduced as the topology becomes resolved (close to $m_{a}=15 \mathrm{GeV}$ ), but the limits are improved by a factor of 10 with respect to the Run 1 result [22].

Another recent result carried out by ATLAS is the search for Higgs boson decays into a pair of $a$ bosons in the $b b \mu \mu$ final state [23], performed using pp collisions data at $\sqrt{s}=13 \mathrm{TeV}$ with an integrated luminosity of $36.1 \mathrm{fb}^{-1}$. The $a \rightarrow b b$ requirement ensures a large branching fraction, while the clean di-muon signature, which can be used for triggering and precision mass reconstruction, helps to suppress background. The search for a narrow di-muon resonance is performed over the range $18 \mathrm{GeV} \leq m_{\mu \mu} \leq 62 \mathrm{GeV}$ and no significant excess of the data above the SM prediction is observed. Upper limits are set on $\left(\sigma_{H} / \sigma_{S M}\right) \times B(H \rightarrow a a \rightarrow b b \mu \mu)$ and are shown in Figure 4 (right).

\section{Conclusions}

The most recent searches for rare and exotic decays of the $125 \mathrm{GeV}$ Higgs boson performed with data collected by the CMS and ATLAS experiments at $\sqrt{s}=13 \mathrm{TeV}$ have been presented. No significant deviations from SM predictions have yet been observed. Nevertheless, the physics potential of the $13 \mathrm{TeV}$ dataset has just started to be extracted. The scalar sector of the Standard 

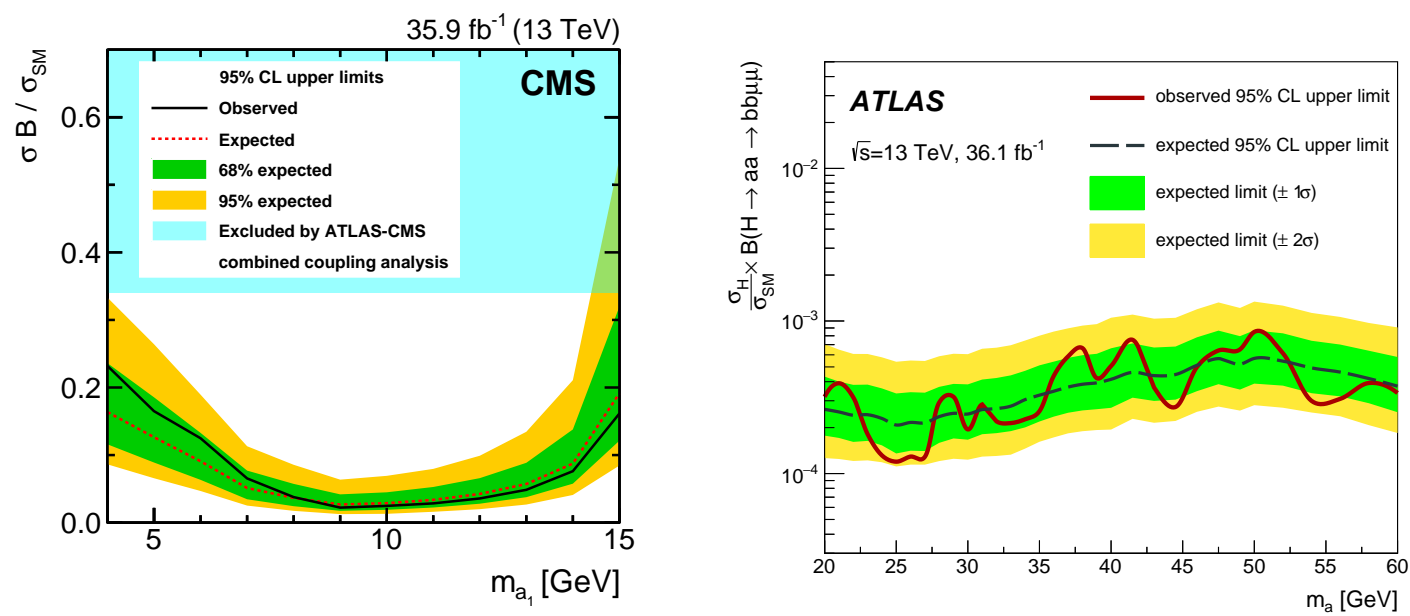

Figure 4: Left: The observed and expected upper limits at $95 \%$ confidence level on the product of signal cross section and the branching fraction $\sigma(p p \rightarrow H+X) \mathscr{B}(H \rightarrow a a) \mathscr{B}^{2}(a \rightarrow \tau \tau)$, relative to the inclusive Higgs boson production cross section $\sigma_{S M}$ predicted in the SM, measured by CMS [21]. Right: The observed and expected upper limits at the $95 \%$ confidence level on $\mathscr{B}(H \rightarrow a a \rightarrow b b \mu \mu)$ given the SM Higgs boson production cross-section in the ggF, VBF and VH modes, measured by ATLAS [23].

Model remains a favoured place to look for new physics effect and LHC data are still sensitive to some BSM theoretical models.

\section{References}

[1] ATLAS Collaboration, "Observation of a new particle in the search for the Standard Model Higgs boson with the ATLAS detector at the LHC", Phys. Lett. B 716 (2012) 1, doi:10.1016/j.physletb.2012.08.020, arXiv:1207.7214.

[2] CMS Collaboration, "Observation of a new boson at a mass of $125 \mathrm{GeV}$ with the CMS experiment at the LHC", Phys. Lett. B 716 (2012) 30, doi:10.1016/j.physletb.2012.08.021, arXiv:1207.7235.

[3] CMS Collaboration, "Observation of a new boson with mass near $125 \mathrm{GeV}$ in pp collisions at $\sqrt{s}=7$ and 8 TeV", JHEP 06 (2013) 081, doi:10.1007/JHEP06(2013)081, arXiv:1303.4571.

[4] ATLAS and CMS Collaborations, "Measurements of the Higgs boson production and decay rates and constraints on its couplings from a combined ATLAS and CMS analysis of the LHC pp collision data at $\sqrt{s}=7$ and 8 TeV", JHEP 08 (2016) 045, doi:10.1007/JHEP08(2016)045, arXiv:1606.02266.

[5] ATLAS Collaboration, "The ATLAS experiment at the CERN large hadron collider ", JINST 3 (2008) S08003, doi:10.1088/1748-0221/3/08/S08003.

[6] CMS Collaboration, "The CMS experiment at the CERN LHC", JINST 3 (2008) S08004, doi:10.1088/1748-0221/3/08/S08004.

[7] ATLAS Collaboration, "Searches for exclusive Higgs and Z boson decays into $J / \psi \gamma, \psi(2 S) \gamma$, and $\Upsilon(n S) \gamma$ at $\sqrt{s}=13 \mathrm{TeV}$ with the ATLAS detector", Phys. Lett. B 786 (2018) 134,

doi:10.1016/j.physletb.2018.09.024, arXiv:1807.00802 
[8] CMS Collaboration, "Search for Higgs and Z boson decays to $J / \psi$ or $\Upsilon$ pairs in the four-muon final state in proton-proton collisions at $\sqrt{s}=13 \mathrm{TeV}$ ", Phys. Lett. B 797 (2019) 134811, doi:10.1016/j.physletb.2019.134811, arXiv:1905.10408

[9] CMS Collaboration, "Search for lepton-flavour-violating decays of the Higgs boson", Phys. Lett. B749(2015) 337, doi:10.1016/j.physletb.2015.07.053, arXiv:1502.07400.

[10] CMS Collaboration, "Search for lepton flavour violating decays of the Higgs boson to $e \tau$ and $e \mu$ in proton-proton collisions at $\sqrt{s}=8 \mathrm{TeV}$ ", Phys. Lett. B763(2016) 472 , doi:10.1016/j.physletb.2016.09.062, arXiv:1607.03561.

[11] ATLAS Collaboration, "Search for lepton-flavour-violating $H \rightarrow \mu \tau$ decays of the Higgs boson with the ATLAS detector", JHEP 11 (2015) 211, doi:10.1007/JHEP11(2015)211, arXiv:1508.03372.

[12] ATLAS Collaboration, Search for lepton-flavour-violating decays of the Higgs and Z bosons with the ATLAS detector, Eur. Phys. J. C 77 (2017) 70, doi:10.1140/epjc/s10052-017-4624-0, arXiv:1604.07730.

[13] ATLAS Collaboration, "Searches for lepton-flavour-violating decays of the Higgs boson in $\sqrt{s}=13$ TeV pp collisions with the ATLAS detector", Submitted to PLB, arXiv:1907.06131.

[14] CMS Collaboration, "Search for lepton flavour violating decays of the Higgs boson to $\mu \tau$ a $e \tau$ in proton-proton collisions at $\sqrt{s}=13 \mathrm{TeV}$, JHEP 06 (2018) 001, doi:10.1007/JHEP06(2018)001, arXiv: 1712.07173.

[15] ATLAS Collaboration, "Combination of Searches for Invisible Higgs Boson Decays with the ATLAS Experiment", Phys. Rev. Lett. 122 (2019) 231801, doi:10.1103/PhysRevLett.122.231801, arXiv:1904.05105.

[16] CMS Collaboration, "Search for invisible decays of a Higgs boson produced through vector boson fusion in proton-proton collisions at $\sqrt{s}=13 \mathrm{TeV}$ ", Phys. Lett. B 793 (2019) 520, doi:10.1016/j.physletb.2019.04.025, arXiv:1809.05937.

[17] CMS Collaboration, "First constraints on invisible Higgs boson decays using $t \bar{t} H$ production at $\sqrt{s}=$ 13 TeV", CMS-PAS-HIG-18-008, url:https://cds.cern.ch/record/2668677.

[18] CMS Collaboration, "Search for Dark Photons in ZH Decays at $\sqrt{s}=13 \mathrm{TeV}$ ", CMS-PAS-EXO-19-007, url:http://cds.cern.ch/record/2674966

[19] ATLAS Collaboration, available at https://atlas.web.cern.ch/Atlas/GROUPS/PHYSICS/PUBNOTES/ATL-PHYS-PUB-2018045/fig_03.png.

[20] CMS Collaboration, available at https://twiki.cern.ch/twiki/pub/CMSPublic/SummaryResultsHIG/run2_plot_BRaa_Type2_tanbeta2.png.

[21] CMS Collaboration, "Search for light pseudoscalar boson pairs produced from decays of the $125 \mathrm{GeV}$ Higgs boson in final states with two muons and two nearby tracks in pp collisions at $\sqrt{s}=13 \mathrm{TeV}$ ", Submitted to PLB, arXiv:1907.07235.

[22] CMS Collaboration, "Search for a very light NMSSM Higgs boson produced in decays of the 125 $\mathrm{GeV}$ scalar boson and decaying into $\tau$ leptons in pp collisions at $\sqrt{s}=8 \mathrm{TeV}$ ", JHEP01(2016)079, doi:10.1007/JHEP01(2016)079, arXiv:1510.06534.

[23] ATLAS Collaboration, "Search for Higgs boson decays into a pair of light bosons in the $b b \mu \mu$ final state in pp collision at $\sqrt{s}=13 \mathrm{TeV}$ with the ATLAS detector", Phys. Lett. B 790 (2019) 1 , doi:10.1016/j.physletb.2018.10.073, arXiv:1807.00539. 